\title{
Design and experimental evaluation of a low-complexity spatial combiner for LTE distributed antenna systems
}

\author{
Josep Soler-Garrido, Magnus Sandell", Filippo Tosato, David Milford and Imran Ahmed
}

\begin{abstract}
This article presents a distributed antenna system (DAS) architecture for small-cell base stations (BTSs), whereby cooperation between the DAS infrastructure and the BTS allows for an increase in performance compared to conventional systems, while at the same time keeping complexity and cost at low levels. Specifically, the article investigates the improvements in uplink physical layer performance achieved by adding an initial antenna combining step in the DAS system before conventional combining and equalization at the BTS. This initial step can be implemented in a very low-complexity fashion by performing all operations in the time domain and using channel state information calculated at the BTS itself. The article presents this technique in the context of an LTE DAS system. Results from both a software simulator and a custom-made hardware prototype are presented, establishing the feasibility of the proposed architecture.
\end{abstract}

\section{Introduction}

In recent times, multiple antenna systems have been one of the main technological drivers for the evolution of wireless communications. In particular, in cellular systems multiple-input multiple-output (MIMO) techniques have successfully been used to increase cell throughput, link capacity, and/or quality [1-3]. Current network deployments typically resort to the use of distributed radio units (RUs) in order to improve the service provided. The socalled remote radio heads containing antennas and RF front-ends but very little processing power $[4,5]$ are strategically placed to optimize coverage. These are typically connected to a central unit or base station (BTS) via fibre-optical links, and can significantly increase system capacity and coverage [6]. These distributed antenna systems (DASs) have been proposed as a cheaper alternative to deploying multiple BTSs, for example, in an indoor space to improve coverage $[7,8]$.

In conventional deployments, BTS and DAS are separate subsystems in the overall network. The DAS can be seen as a fixed infrastructure which routes the signal to and from a third party BTS. Its cost is typically related to

\footnotetext{
*Correspondence: magnus.sandell@toshiba-trel.com

Toshiba Research Europe Ltd., Telecommunications Research Laboratory, 32 Queen Square, Bristol, UK
}

the area that has to be covered. The BTS itself can have different capabilities which depend on the requirements, e.g., total capacity, of the particular deployment. In general, larger deployments in terms of area do not necessarily require a more powerful BTS, especially if the number of users, or their throughput requirements, are low.

The DAS architecture considered in this article is shown in Figure 1, where a coverage extension network is deployed within a specific area, e.g., a building. The system consists of multiple RUs which contain the radio frequency (RF) and antenna elements, and connect to a central hub unit (HU), whose original purpose is to interface between the multiple RUs and the BTS. Given the potentially large number of RUs, this usually requires combining multiple RU signals in the uplink into a number of outputs that is manageable by the BTS, and vice versa for the downlink, where a small number of signals from the BTS are distributed to multiple RUs. Note that the HU may be colocated with the serving BTS, and cooperation between them can provide certain advantages. This is specially the case in deployments where cost is critical and a large number of RUs have to be served by a small-cell BTS with a small number of antenna ports. The availability of many RUs attached to an HU ensures a

\section{照 Springer}

(c) 2013 Soler-Garrido et al.; licensee Springer. This is an Open Access article distributed under the terms of the Creative Commons Attribution License (http://creativecommons.org/licenses/by/2.0), which permits unrestricted use, distribution, and reproduction in any medium, provided the original work is properly cited. 


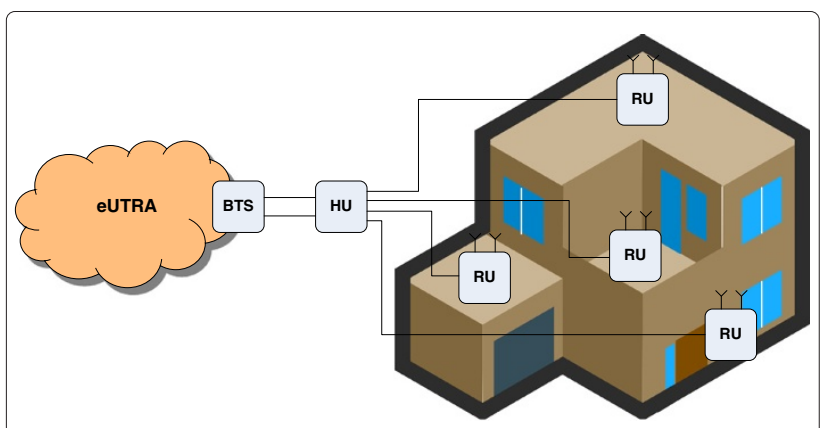

Figure 1 Distributed Antenna System (DAS).

high degree of spatial gain and coverage in the system, but the BTS may be unable to exploit it sufficiently. Conventionally, the signals from all RUs are analogue combined in a noncoherent fashion at the HU before conversion [8]. Even if signals to/from different RUs could be handled independently, a typical small BTS subsystem connected to the HU may only support up to two antenna ports. This article presents an adaptive DAS system which makes use of information fed back from the BTS in order to improve performance and coverage. In the proposed system, the HU itself implements a low-complexity coherent combination of all its input signals. This results in the subsequent BTS being only required to handle a small set of inputs. This approach translates into much lower equipment costs, especially in larger deployments requiring a high number of remote units, but where cost or capacity requirements make the use of multiple BTSs with a high number of antenna ports impractical.

Specifically, we present a two-stage coherent combining scheme where the two combiners serve different purposes and jointly exploit the gain offered by a potentially very large number of RUs. Furthermore, in order to keep complexity and bill of materials low on the DAS infrastructure side, we explore the possibility to perform the combining stage at the HU only in the spatial domain. Thereafter, the second stage at the BTS performs a conventional frequency selective spatial equalization. Moreover, the combining weight calculation at the $\mathrm{HU}$ is assisted by existing channel estimation capabilities at the BTS, reducing complexity further. The HU only performs a simple linear combining operation which does not require the provision of expensive FPGAs or digital signal processors.

Our two-stage DAS concept is applied to a 3GPP LTE Release 8 [9] uplink communication setup as an example. The main deployment scenarios we envisage for our architecture are those typical for DAS systems, i.e., indoor office or residential building, and up to small urban cells, where coverage extension is sought without deploying expensive and heavy-maintenance BTS equipments but rather by using inexpensive DAS infrastructure.
The main contributions of this article are

- We provide a description of a DAS for uplink LTE. A (potentially) large number of remote antennas are connected to an $\mathrm{HU}$ which processes the signals before passing them on to a BTS. Despite the reduced number of BTS antenna ports, the system is able to exploit the spatial gain provided by the multiple RUs.

- As a further simplification, we present the calculation of space-only combiner weights, such that the amount of synchronization, and domain conversions required at the DAS hardware are kept to a minimum.

- A hardware test-bed has been developed in order to prove the concept. Measurements using a hardware channel emulator allow for a direct comparison between different system architectures, and it is shown that the proposed DAS modifications offer a significant benefit over conventional systems.

The remainder of the article is organized as follows. A system overview of our DAS architecture and motivation for the choice of the two-stage combiner are given in Section 2. The problem of computing the combiner weights is addressed in Section 3 and simulation results are shown in Section 4. The hardware setup and measurement results are presented in Section 5, and conclusions are drawn in Section 6.

\section{DAS uplink combining}

As aforementioned, a key advantage of DAS is that geographically distributed RUs can be connected to a single serving BTS in order to exploit the spatial gain. This can particularly be useful when small cells (and including femto- or pico-cells) are deployed in indoor spaces to improve coverage and combat interference. The small size and irregular shape of a small cells make the use of RUs very effective in extending/improving coverage without generating too much interference on adjacent cells. Besides, significant gains can be exploited to boost the received signal without increasing the transmit power.

Although any number of antennas can be used, a practical problem is that existing small-cell BTSs have much more limited capability compared to macrocell BTSs and may not support more than two antenna ports; hence, the challenge is to use a potentially large number $N$ of antennas in a resource-constrained $\mathrm{HU}$ where a very limited amount of processing can be done. If cost is to be kept low, implementation of functions such as channel estimation may not be feasible.

Moreover, because of the frequency selectivity of a wireless channel, an optimum combiner should apply different weights for different frequencies; in practice the smallest frequency unit for which a different set of weights can be 
calculated is a physical resource block (RB). In this case too, a frequency selective combiner for an OFDM-based system requires substantial processing in the frequency domain which entails a range of relatively complex operations such as discrete Fourier transforms (DFT-IDFT pair), synchronisation, frequency offset compensation, etc. In particular, if the objective of the combiner is that of minimising the mean squared error of the useful signal estimate in the presence of unknown interference, additional estimates of the auto- and cross-correlation are required and a matrix inversion operation is necessary to compute the minimum mean-squared error (MMSE) combiner weights for each active RB. In order to avoid placing such high processing demands on the DAS system, we propose the use of space-only weights at the HU, e.g., one set of adaptive weights which applies to all RBs.

Of course, performance of such a simple combiner will be degraded in highly frequency selective channels, especially when using large RB allocations. Simulations and experimental results presented in this article aim to analyse this. It should also be noted that in the multiuser case, the radio resource manager (RRM) at the BTS has to be aware of the DAS combining scheme in order to assign entire subframes to users, or group those with similar propagation conditions, so that they share the available bandwidth at any time instant. In the article, we present single-user results intended to quantify the potential performance improvements at physical layer attainable by the proposed DAS solution, without going into RRM implementation details.

This solution is effectively a two-stage combiner. Assuming two antenna ports at the BTS, an $\mathrm{N}$-input 2output spatial combiner at the HU, whose aim is to exploit the spatial gain offered by coherent combining of the $N$ RUs is followed by a conventional 2-input 1-output MMSE space-frequency combiner at the BTS, whose main task is to combat noise and unknown interference. This separation of roles between the two combiners is motivated by the choice of a frequency non-selective combiner at the HU. In fact, the limitation of having a single set of weights at the $\mathrm{HU}$ for the whole configured bandwidth implies that the HU combiner may have poor noise and interference rejection capabilities if the channel and/or interference are frequency selective across the used spectrum. In this respect, the use of a wideband MMSE solution for the $\mathrm{HU}$ combiner is ineffective and may give poor results in most channel conditions as the matrix inversion operation tends to amplify the mismatch between combiner weights and actual auto- and crosscovariances values on each RB. Therefore, our choice for the inner combiner is a simple maximal ratio combiner (MRC).

Of course, in order to calculate weights for the outer $\mathrm{HU}$ combiner using channel estimates at the BTS, the latter must have direct (noncombined) visibility of the different
$N$ inputs at the HU. To allow this, our solution is to cyclically switch between the $N$ antennas at the HU and feed its signal through to one of the input ports on the BTS. At the same time, the $N$ antennas are combined and the combined signal is passed on to the BTS through the other input port. By doing so, the channel can be estimated periodically for each of the $N$ RU. In the next section, we motivate our choice of average MRC weights applied across the bandwidth.

The DAS with the proposed BTS and HU is outlined in Figure 2. The switch at the HU selects one antenna signal and feeds it through to the BTS. Since each individual antenna is observed at the BTS every $N$ switching actions, if the HU switches every subframe the channel estimates will never be severely outdated for moderate UE speeds. In fact, one of the channel estimates will be right on time, one will be outdated by one subframe, one will be two subframes old and so forth up to the $N$ th oldest estimate that will experience the largest delay of $N$ subframes.

\section{Combiner weights}

As mentioned in the previous section, at the $\mathrm{HU}$, it is desirable to use the same weights for all subcarriers for reasons of complexity. Since MRC cannot be performed per subcarrier, the weights must be chosen with a global cost function, i.e., include all subcarriers. The received signal at the HU with $N$ antennas is

$$
r_{n, k}=h_{n, k} x_{k}+v_{n, k}
$$

where $r_{n, k}$ is the received signal, $h_{n, k}$ is the channel, $x_{k}$ is the transmitted signal, $v_{n, k}$ is the additive noise and $n$ and $k$ denote the antenna and subcarrier, respectively. If the weights $w_{n}^{*}$ are used to form the combined output

$$
y_{k}=\sum_{n=0}^{N-1} w_{n}^{*} r_{n, k}=\sum_{n=0}^{N-1} w_{n}^{*} h_{n, k} x_{k}+\sum_{n=0}^{N-1} w_{n}^{*} v_{n, k}
$$

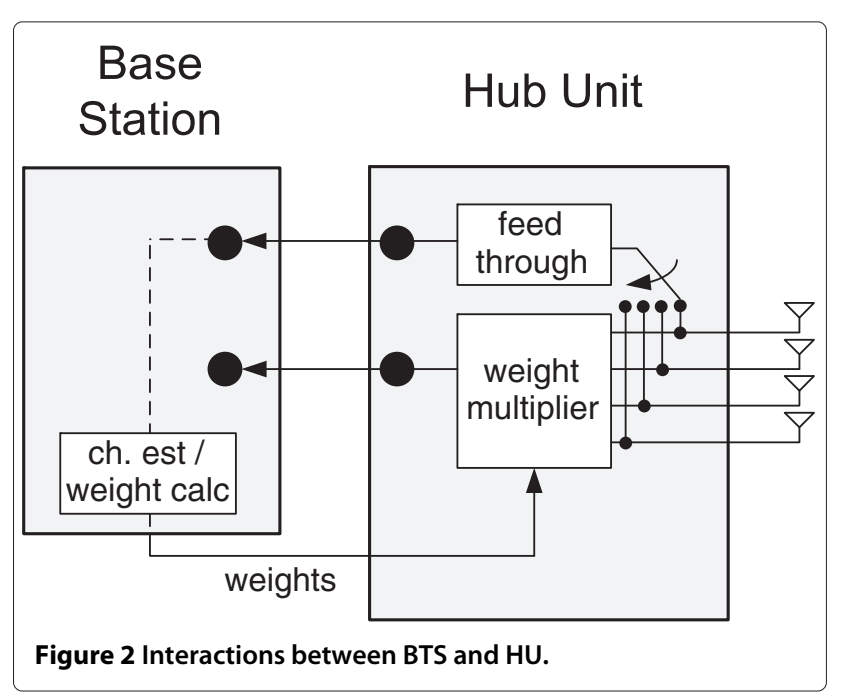


a reasonable choice of metric would be to maximize the combined SNRs over the $K$ subcarriers

$$
\sum_{k=0}^{K-1} \gamma_{k}=\sum_{k=0}^{K-1} \frac{\left|\sum_{n=0}^{N-1} w_{n}^{*} h_{n, k}\right|^{2} \sigma_{x}^{2}}{\sum_{n=0}^{N-1}\left|w_{n}\right|^{2} \sigma_{v}^{2}}
$$

where $\gamma_{k}$ is the (instantaneous) SNR on the $k$ th subcarrier and $\sigma_{v}^{2}=E\left\{\left|v_{n, k}\right|^{2}\right\}$ is the noise variance.

The sum of SNRs in (3) can be written as

$$
\begin{aligned}
\sum_{k=0}^{K-1} \gamma_{k} & =\frac{\sigma_{x}^{2}}{\sigma_{v}^{2}} \frac{\sum_{k=0}^{K-1} \sum_{n=0}^{N-1} \sum_{n^{\prime}=0}^{N-1} w_{n}^{*} h_{n, k} h_{n^{\prime}, k}^{*} w_{n^{\prime}}}{\sum_{n=0}^{N-1}\left|w_{n}\right|^{2}} \\
& =\frac{\sigma_{x}^{2}}{\sigma_{v}^{2}} \frac{\sum_{n=0}^{N-1} \sum_{n^{\prime}=0}^{N-1} w_{n}^{*}\left(\sum_{k=0}^{K-1} h_{n, k} h_{n^{\prime}, k}^{*}\right) w_{n^{\prime}}}{\sum_{n=0}^{N-1}\left|w_{n}\right|^{2}} .
\end{aligned}
$$

This is a Rayleigh quotient [10], so it is clear that the optimal choice of weights is the eigenvector of $\mathbf{R}$ corresponding to the largest eigenvalue, where $R_{n, n^{\prime}}=$ $\sum_{k=0}^{K-1} h_{n, k} h_{n^{\prime}, k}^{*}$. The correlation matrix can also be computed using the impulse response $g_{n, l}$; if the frequency response is

$$
h_{n, k}=\frac{1}{\sqrt{K}} \sum_{l=0}^{L-1} g_{n, l} e^{-j 2 \pi k l / K}
$$

where $L$ is the number of time-domain taps, the correlation matrix is

$$
\begin{aligned}
R_{n, n^{\prime}} & =\sum_{k=0}^{K-1}\left(\frac{1}{\sqrt{K}} \sum_{l=0}^{L-1} g_{n, l} e^{-j 2 \pi k l / K}\right)\left(\frac{1}{\sqrt{K}} \sum_{l^{\prime}=0}^{L-1} g_{n^{\prime}, l}^{*} l^{j 2 \pi k l^{\prime} / K}\right) \\
& =\sum_{l=0}^{L-1} \sum_{l^{\prime}=0}^{L-1} g_{n, l} g_{n^{\prime}, l^{\prime}}^{*} \sum_{k=0}^{K-1} \frac{1}{K} e^{-j 2 \pi k\left(l-l^{\prime}\right) / K} \\
& =\sum_{l=0}^{L-1} \sum_{l^{\prime}=0}^{L-1} g_{n, l} g_{n^{\prime}, l^{\prime}}^{*} \delta_{l-l^{\prime}}=\sum_{l=0}^{L-1} g_{n, l} g_{n^{\prime}, l}^{*} .
\end{aligned}
$$

However, this involves computing the correlation matrix $\mathbf{R}$ (either in the frequency or time domain) and its largest eigenvector. To reduce complexity further we propose in this article a simpler way of choosing the weights $w_{n}$. The correlation matrix in (6) can be viewed as the sum of that of all $L$ taps; if the first tap $g_{n, 0}$ is strong compared to the others, which can be expected for a DAS system, we may use only that one to estimate the correlation matrix $\mathbf{R}$. In this case

$$
R_{n, n^{\prime}} \approx g_{n, 0} g_{n^{\prime}, 0}^{*} \Rightarrow w_{n}=g_{n, 0} .
$$

The first tap of the impulse response can also be computed as

$$
g_{n, 0}=\frac{1}{\sqrt{K}} \sum_{k=0}^{K-1} h_{n, k}
$$

from the IDFT. Hence, the weights can be computed as a simple average directly from the frequency response $h_{n, k}$ without the need for IDFTs or eigenvalue decomposition.

\section{Simulation results}

The physical layer of LTE was simulated in Matlab using the Winner II channel models [11]. The worst possible scenario considered in terms of delay spread and frequency selectivity is B1 (urban microcell) with nonline-of-sight (NLOS) propagation; it has 20 channel taps and a delay spread of $485 \mathrm{~ns}$. We use the $10 \mathrm{MHz}$ mode, with up to $50 \mathrm{RBs}$ available, although either $10 \mathrm{RB}$ or 40 $\mathrm{RB}$ are considered in the examples provided. The simulations use modulation and coding scheme (MCS) 15, which is 16QAM modulation with a code rate of approximately $1 / 2$. The BTS is assumed to have two input ports and the HU is connected to four single-antenna RUs spaced 10 wavelengths apart, a conservative number as DAS systems are expected to benefit from substantially uncorrelated antennas. We assume that the average received power per RU is the same; this might be considered a worst-case scenario as well, since more gains can be expected with unequal powers, a more realistic scenario. The impact of the approximation (7), introduced in the previous section, is shown in Figure 3 for a single user employing $10 \mathrm{RB}$, where it can be seen to result in

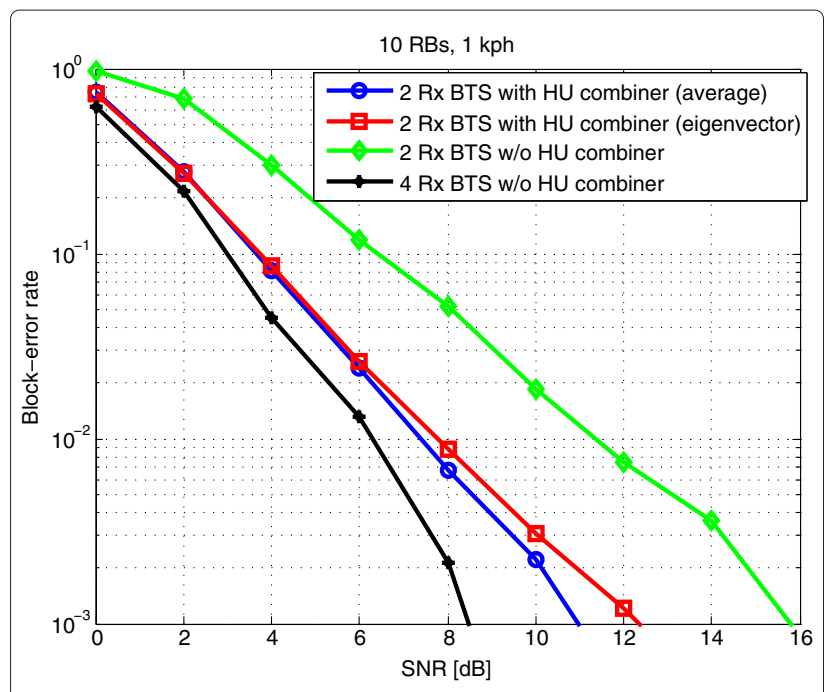

Figure 3 Performance of simplified space-only combiner (10 RBs, $1 \mathrm{~km} / \mathrm{h}$ ). 
negligible degradation. Its performance is compared to a two- and four-antenna BTS without an HU. The latter is included only as an upper limit to what could be achieved with four antennas since we assume there are only two ports on the BTS. As can be seen, our DAS approach with space-only combining at the $\mathrm{HU}$ offers a significant improvement over a system without coherent HU combining. As aforementioned, for space-only combining the number of active RBs is important since the channel effectively gets wider when they increase. In Figure 4, the block-error rate (BLER) is shown when $40 \mathrm{RBs}$ are used. Although the relative performance is slightly degraded, it offers a clear improvement over a pure two-antenna system.

Finally, and before moving on to the experimental results, we analyse the effect of user equipment (UE) speed on performance. This is interesting as the inherent delay on the CSI employed at the HU could render the combiner ineffective in the presence of highly timevarying channels. However, results in Figure 5 show that, at least for the four-antenna case, this is not a critical variable, and the $\mathrm{HU}$ combiner offers acceptable performance even for speeds up to $40 \mathrm{~km} / \mathrm{h}$, reasonable in a urban microcell scenario.

\section{Experimental results}

In this section, a hardware prototype of the described uplink LTE DAS system is presented, along with some experiments aimed at quantifying, in practical scenarios and under realistic propagation conditions, the performance gains that can be attained by the $\mathrm{HU}$ processing described in previous section, and linking them with the simulation results provided.

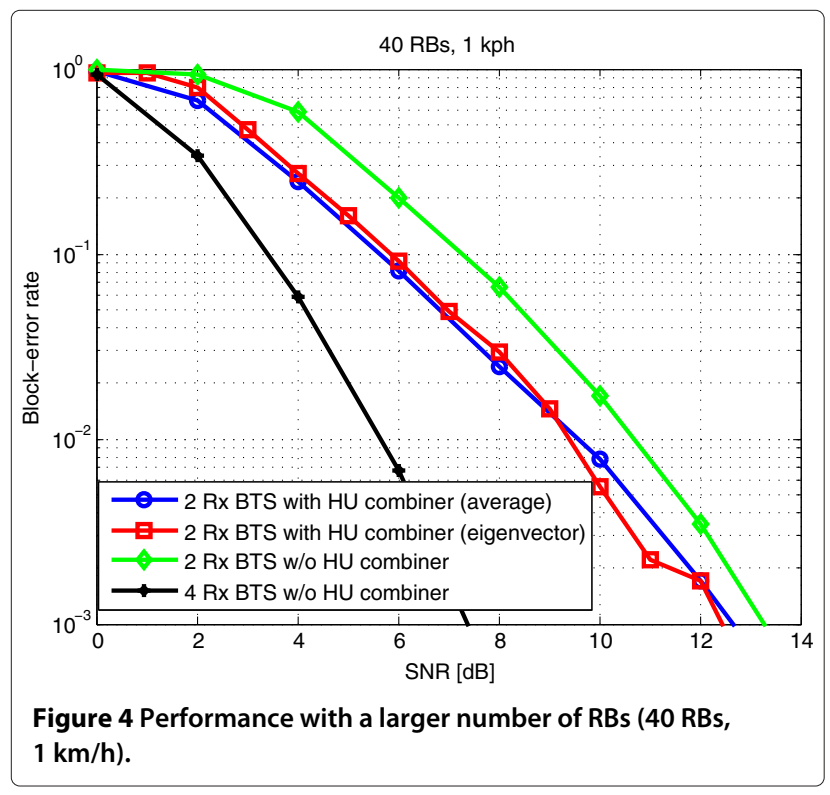

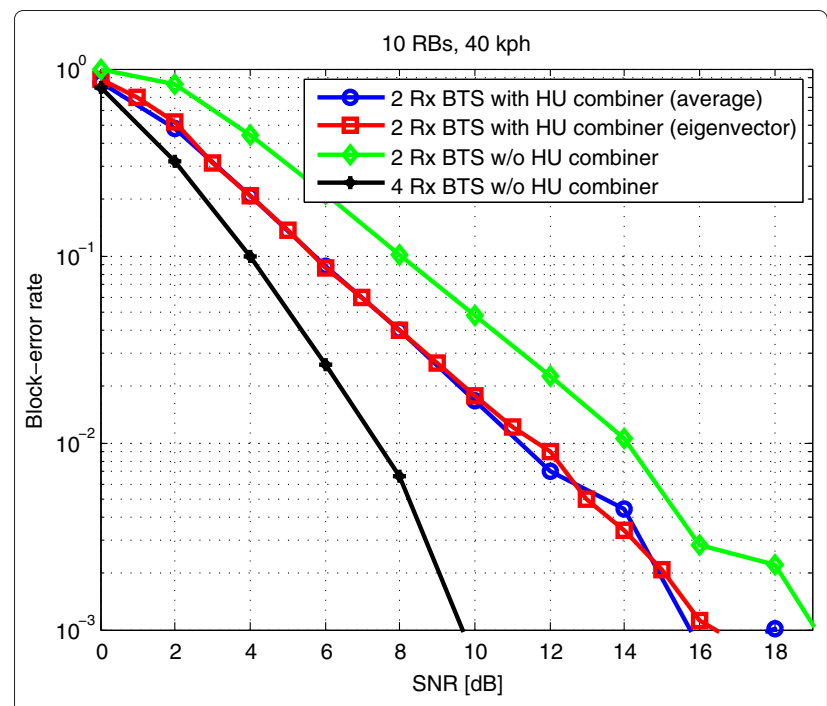

Figure 5 Performance with a larger UE speed (10 RBs, $40 \mathrm{~km} / \mathrm{h})$.

\subsection{Hardware prototype}

The hardware prototype consists of an eNodeB development system, a test mobile terminal, a custom-made RF board performing the functions of four RUs and an FPGA development board performing $\mathrm{HU}$ functions. In order to implement the propagation channel, an Elektrobit C8 hardware emulator is employed. A photograph of the kit is provided in Figure 6.

The eNodeB is a picoChip PC9609 small-cell LTE development system, with a fully featured Rel. 8 PHY. This system contains two antenna ports and operates in 3GPP band 13, i.e., with uplink centred at $782 \mathrm{MHz}$ and downlink at $751 \mathrm{MHz}$. The bandwidth employed is $10 \mathrm{MHz}$. In this case, as we are interested in measuring performance at the physical layer, no protocol stack is employed, using instead a simple PHY driver collecting low level measurements.

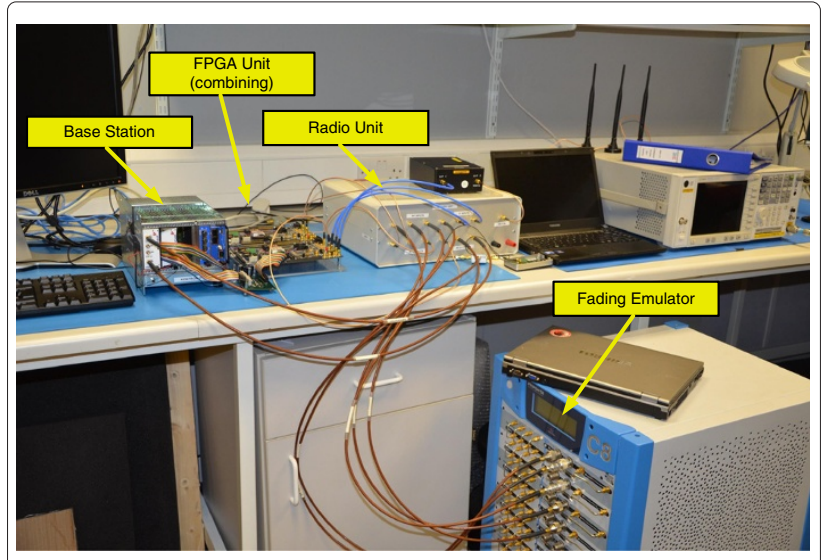

Figure 6 Overview of the experimental setup. 
At the other end of the system lies an Aeroflex TM500 test UE, which again is operated in HARQ only mode, i.e., it establishes only low level communication with the eNodeB. Most of the settings are manually configured and remain static during the experiments, including uplink and downlink resource allocation, MCS values and transmitter power.

An RF board implementing four receivers was built in order to emulate a system with four single-antenna RUs. The basic diagram of this board is depicted in Figure 7. A low-IF architecture is chosen whereby the four independent input (uplink) RF signals are amplified, filtered and downconverted to IF with a centre frequency of $15.36 \mathrm{MHz}$. Thereafter, they are sampled by a transformer coupled ADC for subsequent processing in digital domain. It should be noted that no image-rejection method beyond a simple RF bandpass SAW filter is included on these receivers as they are designed for cabled operation and a single channel at $782 \mathrm{MHz}$ is expected at their input.

The digitized IF signals are processed by two Altera EP1S80 FPGA development boards. These boards actually contain the ADCs from the previous diagram, along with two FPGA devices employed for HU digital signal processing. Since propagation in the prototype is emulated in hardware, and hence all the RUs are colocated and next to the HU, the IF signals can directly be connected to the ADC inputs. Figure 8 shows a simplified $\mathrm{HU}$ processing implementation, where signals are digitally downconverted, with a baseband sampling rate of 15.36 $\mathrm{MHz}$. Two output branches are generated as previously described, with the first one containing a combined signal according to a current set of weights, and the second one being a simple feed-through of one if the inputs, used for training purposes. These two outputs are sent to the inputs of the eNB. It should be noted that in our kit, this

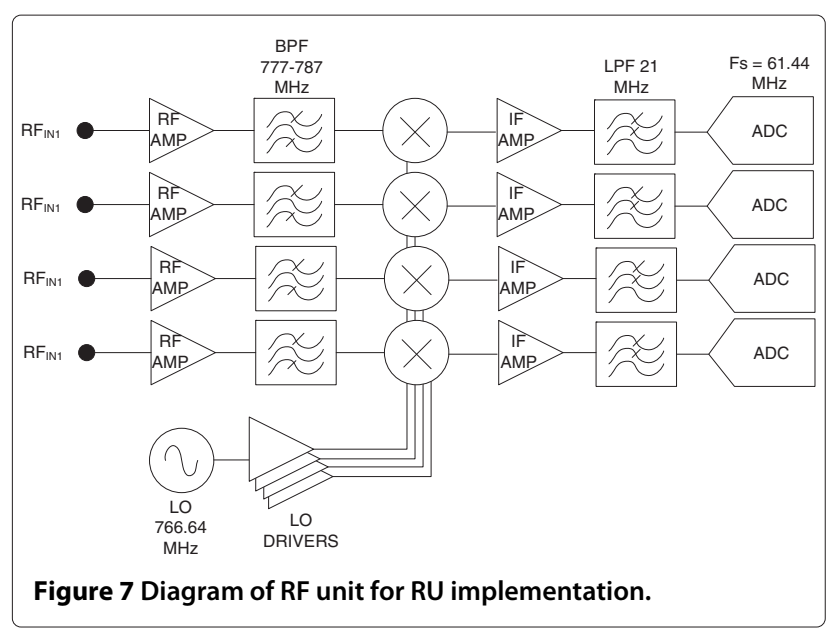

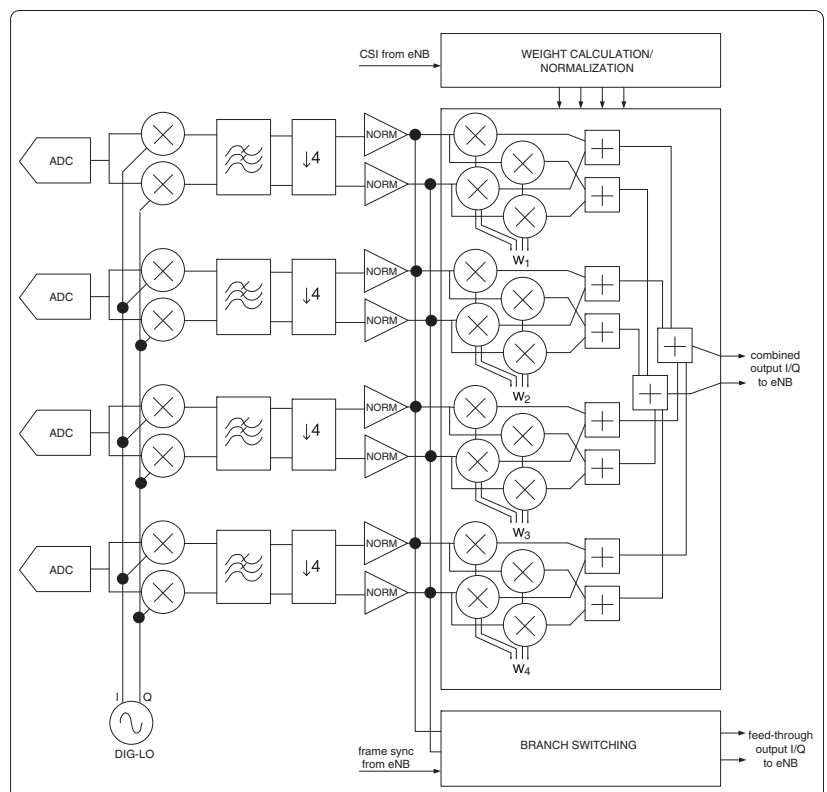

Figure 8 Diagram of hub unit digital implementation.

is done in the RF domain, so subsequent DAC and upconversion stages are required, which are not shown in the diagram. The RF signals from this point onwards are normalized at a constant sufficiently high level for the eNB RF front end.

The weight calculation modules updates the weights every millisecond, but CSI for only one of the four branches is updated on every subframe period. In the prototype, this CSI for the antenna port used for training is sampled at the HU from the digital GPIO outputs at the eNodeB baseband chip. The communication is performed in serial mode, with a single coefficient being sent for each $\mathrm{RB}$ in 32-bit format (I and Q), followed by a single scaler applied at the BTS across the entire bandwidth.

\subsection{Measurements}

In the evaluation setup, the BTS records values of postequalizer SNR. It is always using MRC on its two input ports, regardless of whether these come from two antennas or the HU. In both cases, the SNR is calculated by the BTS in a conventional manner by using the MIMO channel estimate, the MRC coefficients and the noise power estimate. The measured results are averaged, and captured at a rate of approximately 3 per second. The results presented compare the distribution of measured SNR for the cases of either a two fixed antenna case or a space-only MRC combiner at the HU. The channel emulator is programmed with the channel taps corresponding to scenario B1 NLOS of the Winner II model [11]. Parameters such as transmit power and uplink MCS are selected in order to achieve a midrange average SNR. Variations on SNR 
are entirely due to random fading, which in this case is uncorrelated for all the antennas.

The first empirical distribution measured, for the case of $10 \mathrm{RB}$ and $1 \mathrm{~km} / \mathrm{h}$ user speed is shown in Figure 9. A clear performance improvement is observed for the HU-combiner case, increasing the measured average SNR from $14.2 \mathrm{~dB}$ in the two-antenna case to approximately $16.2 \mathrm{~dB}$, i.e., a postequalization gain of $2 \mathrm{~dB}$. Looking at the left-hand tail of the distributions, a substantial reduction in outage probability can also be intuitively expected. These results can also be linked to the previous simulated ones. The measurements correspond to SNR at the input of the channel decoder, so given the simulated performance curves for the LTE turbo code at different rates, it is possible to map each measured SNR sample to an expected error rate, and then compute an average. For the presented distribution, an error rate of $3.2 \times 10^{-2}$ is obtained for the no HU-combiner case, which is improved to approximately $4.5 \times 10^{-3}$ for the combined case. Looking at Figure 3, the measurements are consistent with simulated ones at an input SNR level of between 9 and $10 \mathrm{~dB}$.

The second set of results measure the reduction in gain when moving to $40 \mathrm{RBs}$. Figure 10 shows the empirical distribution. In this case, the gap between the two empirical distributions is reduced, but gains are still noticeable. The HU combiner is now able to increase average SNR from $14.2 \mathrm{~dB}$ to $15.8 \mathrm{~dB}$. In terms of expected BLER, the distributions shown yield approximately $1.5 \times 10^{-2}$ for the no combiner case and $6.0 \times 10^{-3}$ for the HU combiner, which are again consistent with simulated results on Figure 4 at a similar SNR range, in fact indicating a slightly lower than expected BLER in the measured results when

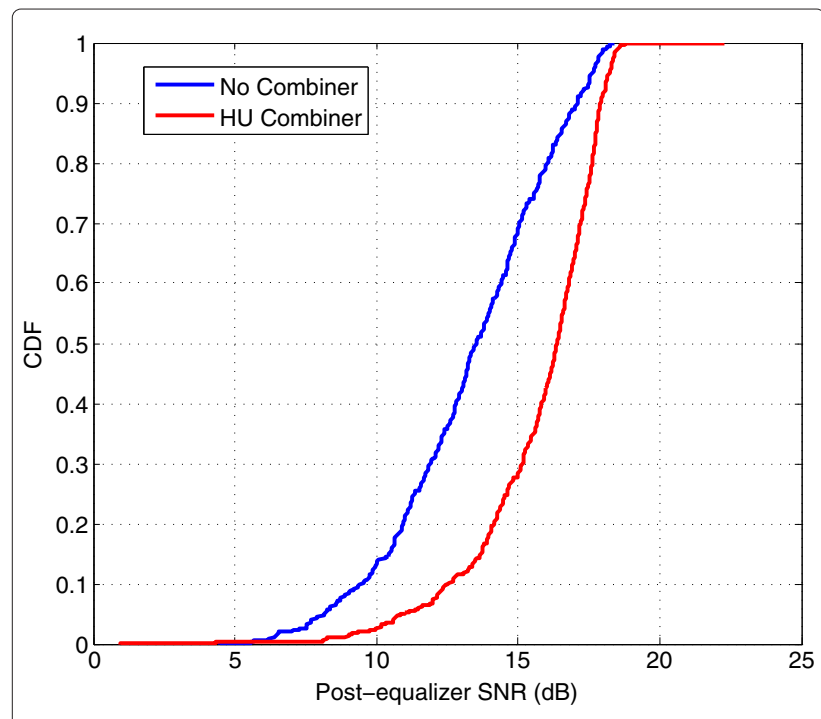

Figure 9 Measured distributions for $10 \mathrm{RB}$ and $1 \mathrm{~km} / \mathrm{h}$ UE speed.

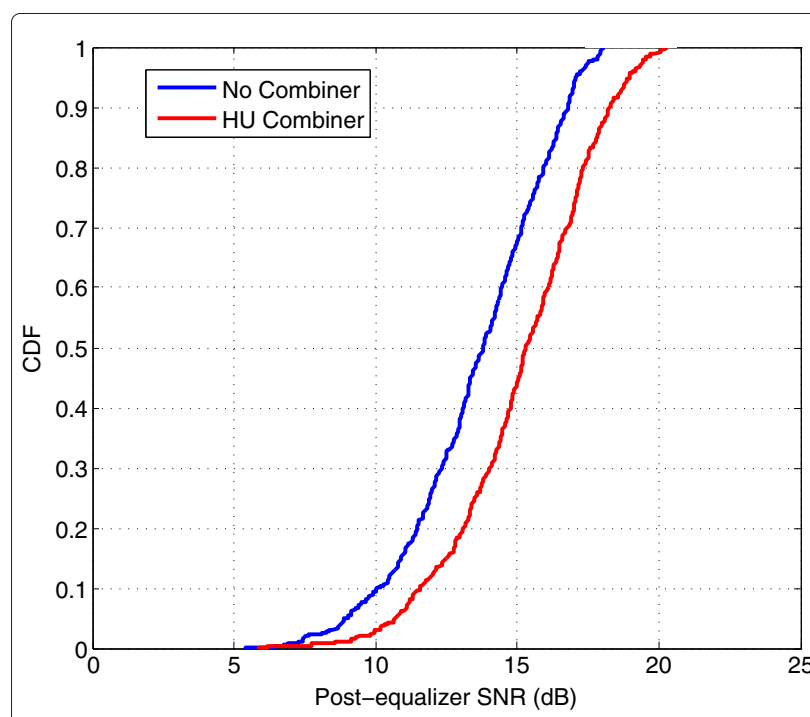

Figure 10 Measured distributions for $40 \mathrm{RB}$ and $1 \mathrm{~km} / \mathrm{h}$ UE speed.

the HU combiner is enabled. Such small variations could be attributed to, e.g., differences between the simulated channel model and the fixed-point one implemented on the $\mathrm{C} 8$, which only approximates the delays of the taps in order to reduce hardware resources.

Finally, measured results for different speeds are provided in order to show the robustness of the method to high mobility scenarios. Figure 11 shows measured average SNR and expected block error rates for the previous $10 \mathrm{RB}$ case at 1, 5, 20 and $40 \mathrm{~km} / \mathrm{h}$. As can be observed, the slight CSI latency in the HU combiner has no significant impact on performance at moderate speeds typical of the chosen scenario, confirming previous simulation results.

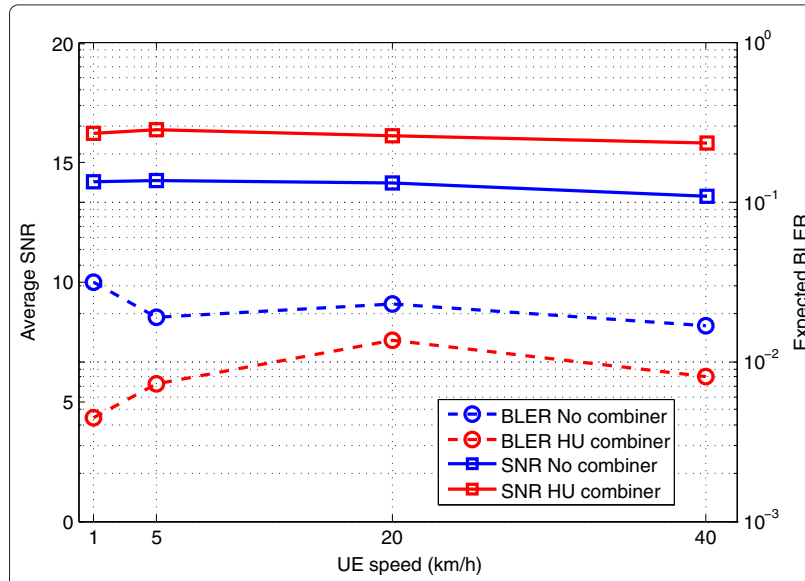

Figure 11 Comparison of measured average SNR and expected BLER at different speeds. 


\section{Conclusions}

In this article, we propose a DAS architecture suitable for small-cell BTSs. It provides a low-complexity solution able to make better use of the spatial gain in the uplink of DAS while still employing low-cost BTS hardware. This is achieved by introducing an initial combining stage at the HU, where signals from different RUs are coherently combined. The article explores the performance of a space-only combiner where the uplink SNR is enhanced by applying a single set of weights across all frequencies at the HU. These results are backed by measurements from a hardware prototype making use of a precommercial Rel. 8 eNodeB, a custom-made RF and digital baseband combiner setup, a test LTE UE and a hardware channel emulator. The experimental results show clear performance gains for the combiner case even when the average received power is the same for all RUs. Moreover, the measurements are in line with simulated results, establishing the feasibility of the proposed architecture in practical real-life deployment in the presence of practical hardware impairments and realistic propagation conditions.

\section{Competing interests}

The authors declare that they have no competing interests.

\section{Acknowledgements}

The authors would like to thank the directors at Toshiba's Telecommunication Research Laboratory in Bristol for their constant support. They would also like to acknowledge the support of the Wireless Systems Laboratory at Toshiba's

Corporate R\&D Center, and especially the assistance provided by Dr.

Tsuguhide Aoki throughout the entire project. They would also like to

acknowledge the work done by Yue Tian during his MSc internship at Toshiba

TRL supporting this project.

Received: 29 September 2012 Accepted: 18 February 2013

Published: 14 March 2013

\section{References}

1. Q Li, G Li, W Lee, M Lee, D Mazzarese, B Clerckx, Z Li, MIMO techniques in WiMAX and LTE: a feature overview. IEEE Commun. Mag. 48(5), 86-92 (2010)

2. J Andrews, W Choi, R Heath, Overcoming interference in spatial multiplexing MIMO cellular networks. IEEE Wirel. Commun. Mag. 14(6), 95-104 (2007)

3. $\mathrm{QH}$ Spencer, AL Swindlehurst, M Haardt, Zero-forcing methods for downlink spatial multiplexing in multiuser MIMO channels. IEEE Trans. Signal Process. 52(2), 461-471 (2004)

4. SG Park, BH Ryu, DY Kim, in Proceedings of IEEE International Conference on ICT Convergence (ICTC). Uplink multi-point reception effect using aggregate base station architecture (Seoul, Korea, 2011), pp. 172-176

5. S Pato, J Pedro, J Santos, A Arsénio, P Inácio, P Monteiro, in Proceedings of the Instituto de Telecomunicações Conference on Telecommunication (ConfTele). On building a distributed antenna system with joint signal processing for next-generation wireless access networks: the FUTON approach (Santa Maria de Feira, Portugal, 2009)

6. H Li, J Hajipour, A Attar, V Leung, Efficient HetNet implementation using broadband wireless access with fiber-connected massively distributed antennas architecture. IEEE Wirel. Commun. 18(3), 72-78 (2011)

7. MJ Crisp, S Li, A Watts, RV Penty, IH White, Uplink and downlink coverage improvements of $802.11 \mathrm{~g}$ signals using a distributed antenna network. J. Lightw. Technol. 25(11), 3388-3395 (2007)

8. D Wake, A Nkansah, NJ Gomes, Radio over fiber link design for next generation wireless systems. J. Lightw. Technol. 28(16), 2456-2464 (2010)
9. 3GPP TR 36211 v890, E-UTRA physical channels and modulation (2009) [http://www.3gpp.org/ftp/Specs/archive/36_series/36.211/36211-890. zip]

10. G Strang, Linear Algebra and Its Applications, 3rd edn. (Harcourt Brace Jovanovich, San Diego, USA, 1986)

11. P Kyösti, J Meinilä, L Hentilä, X Zhao, T Jämsä, C Schneider, M Narandzic, M Milojevic, A Hong, J Ylitalo, VM Holappa, M Alatossava, R Bultitude, Y de Jong, T Rautiainen, WINNER II channel models (2007). [http://www.istwinner.org/WINNER2-Deliverables/D1.1.2v1.1.pdf]

doi:10.1186/1687-1499-2013-69

Cite this article as: Soler-Garrido et al:: Design and experimental evaluation of a low-complexity spatial combiner for LTE distributed antenna systems. EURASIP Journal on Wireless Communications and Networking 2013 2013:69.

\section{Submit your manuscript to a SpringerOpen ${ }^{\mathcal{O}}$ journal and benefit from:}

- Convenient online submission

- Rigorous peer review

- Immediate publication on acceptance

- Open access: articles freely available online

- High visibility within the field

- Retaining the copyright to your article

Submit your next manuscript at $>$ springeropen.com 